Pacific Journal of Mathematic 


\title{
COMPLETENESS PROPERTIES FOR CONVERGENCE SPACES
}

\section{E. LOWEN-COLEBUNDERS}

\begin{abstract}
For uniform convergence spaces a completeness concept has been introduced by Cook and Fisher. For a uniformizable convergence space it is of interest whether among the uniform convergence structures inducing the given structure, one can find a complete one. Keller has shown that this is the case for every Hausdorff convergence space. Our aim is to introduce a stronger completeness concept. We have developed a theory in which completeness is a generalization of topological completeness for metrizable spaces.
\end{abstract}

We have based our theory on various completeness properties which have been introduced for topological spaces such as subcompactness, basecompactness and cocompactness [13], [22], [1], [3], [4]. A survey of those topological completeness properties is given by Aarts and Lutzer in [6].

The crucial point in the introducing of completeness for convergence spaces was the definition of the concept of pointbases which for a convergence space plays the role of the fundamental systems of neighborhoods of a topology. Using this notion we shall introduce and study two completeness concepts.

The theory developed here corresponds to a part of the authors Thesis [19].

For all notational conventions we refer to [7] and [12]. Definitions on convergence spaces that are used can be found in [16] and [17]. We recall those being used frequently.

Let $X$ be a set and $x \in X$. The filter generated by $\{x\}$ is denoted by $\dot{x}$. If $\mathscr{G} \subset 2^{X}$ is a nonempty family with the finite intersection property, then $[\mathscr{G}]$ stands for the filter generated by $\mathscr{G}$.

A convergence space $(X, q)$ is a set $X$ together with a map $q$ which to any point $x \in X$ assigns a family $q x$ of filters on $X$. The filters in $q x$ are said to converge to $x$. For every $x \in X, q x$ contains $\dot{x}$ and for every filter $\mathscr{F}$ in $q x$ the filter $\mathscr{F} \cap \dot{x}$ as well as every filter finer than $\mathscr{F}$ belongs to $q x$.

If in addition for every $x \in X$ the intersection of all the filters in $q x$ belongs to $q x$ then the convergence space is said to be pretopological.

With a convergence space $(X, q)$ one associates a pretopological respectively topological modification denoted by $(X, \psi q)$ respectively $(X, \lambda q)[16]$. 
If $(X, q)$ is a convergence space and $Y$ is a subset on $X$ then $q_{Y}$ denotes the structure on $Y$ induced by $q$. If $\mathscr{F}$ is a filter on $X$ then $\alpha(\mathscr{F})$ stands for its adherence in $(X, q)$.

\section{Pointbase.}

1.1. Definition. Let $X$ be a convergence space. We call a family $\mathscr{A}$ of subsets of $X$ a pointbase for $X$ if for each point $x \in X$ we can find a filterbase $\mathscr{A}(x) \subset \mathscr{A}$ such that the following conditions are satisfied

(1) $\mathscr{A}(x) \subset \dot{x}$.

(2) For a filter $\mathscr{F}$ converging to $x$ we have that $\mathscr{F} \cap \mathscr{A}(x)$ is a filterbase converging to $x$.

It is clear that any family of subsets of $X$ containing a pointbase is itself a pointbase.

In case the convergence structure is pretopological (in particular topological) the condition in the previous definition can be simplified in the following way.

1.2. Proposition. If $X$ is a pretopological space then a family $\mathscr{A}$ of subsets is a pointbase of $X$ if and only if for each point $x \in X$ we have that $\mathscr{A} \cap \mathscr{B}(x)$ is a base for the neighborhoodfilter $\mathscr{B}(x)$.

Proof. If $\mathscr{A}$ is a pointbase for a pretopological space then it follows from condition (2) in (1.1) that $\mathscr{B}(x) \cap \mathscr{A}(x)$ is a filterbase and $\mathscr{B}(x) \supset$ $[\mathscr{B}(x) \cap \mathscr{A}] \supset[\mathscr{B}(x) \cap \mathscr{A}(x)] \supset \mathscr{B}(x)$. For the converse, suppose that for each $x \in X$ the collection $\mathscr{B}(x) \cap \mathscr{A}$ is a base for $\mathscr{B}(x)$. Then $\mathscr{A}(x)=\mathscr{B}(x) \cap \mathscr{A}$ clearly satisfies the conditions of Definition 1.1.

If for a $T_{1}$ convergence space all its pointbases are given then the space is completely determined as follows from the next result.

1.3. THEOREM. If $(X, q)$ and $(X, p)$ are $T_{1}$ convergence spaces with the same family of pointbases, then $p$ and $q$ coincide.

Proof. We shall show that $p$ is coarser than $q$.

Let $\mathscr{F}$ be a filter converging to a point $x$ in $q$.

Suppose $\mathscr{F}$ does not converge to $x$ in $p$. Then consider the collections $\mathscr{A}(x)=\{A \quad x \in A, A \notin \mathscr{F}\}$ and $\mathscr{A}(y)=\{A \quad y \in A, x \notin A\}$ for $y \neq x$. We shall show that $\mathscr{A}=\cup_{z \in X} \mathscr{A}(z)$ is a pointbase for $p$.

For a filter $\mathscr{G}$ converging to $x$ in $p$ we have that $\mathscr{G} \cap \mathscr{A}(x)$ is a filterbase and that $[\mathscr{G} \cap \mathscr{A}(x)]=\mathscr{G} \cap \dot{x}$. 
For a filter $\mathscr{G}$ converging to $y \neq x$ in $p$ we have that $\mathscr{G} \cap \mathscr{A}(y)$ is a filterbase since $(X, p)$ is $T_{1}$ and that $[\mathscr{G} \cap \mathscr{A}(y)]=\mathscr{G} \cap \dot{y}$.

But then $\mathscr{A}$ also is a pointbase for $(X, q)$. Consequently we can find a filterbase $\mathscr{B}(x) \subset \mathscr{A}$ such that $\mathscr{B}(x) \subset \dot{x}$ and $\mathscr{F} \cap \mathscr{B}(x)$ is a filterbase. Since $\mathscr{B}(x) \subset \mathscr{A}(x)$ we have that $\mathscr{F} \cap \mathscr{A}(x)$ is nonempty, which is impossible.

Without the $T_{1}$ condition the previous theorem is not valid as follows from the following example.

Counterexample. Let $X$ be an infinite set and $x$ and $y$ two different points in $X$. Then consider two different sets $B$ and $C$ both containing $\{x, y\}$.

We define the following pretopologies on $X$ by their neighborhoodfilters

$$
\begin{aligned}
& \mathscr{B}_{q}(x)=[B], \mathscr{B}_{q}(y)=[C], \mathscr{B}_{q}(z)=\dot{z} \text { for } z \neq x \text { and } z \neq y \\
& \mathscr{B}_{p}(x)=[C], \mathscr{B}_{p}(y)=[B], \mathscr{B}_{p}(z)=\dot{z} \text { for } z \neq x \text { and } z \neq y .
\end{aligned}
$$

Clearly $q$ is different from $p$ but they have the same family of pointbases.

2. Completeness. The following definitions are inspired by the notions of cocompactness, basecompactness and subcompactness of topological spaces [1], [3], [22], [13].

2.1. Definition. A convergence structure is cocomplete if it has a pointbase $\mathscr{A}$ with the property that for any subfamily $\mathscr{C} \subset \mathscr{A}$ with the finite intersection property $[\mathscr{C}]$ has a nonempty adherence.

It is then called cocomplete with respect to $\mathscr{A}$.

2.2. Definition. A convergence structure is subcomplete if it has a pointbase $\mathscr{A}$ with the property that any filterbase $\mathscr{B} \subset \mathscr{A}$ has a nonempty adherence.

It is then called subcomplete with respect to $\mathscr{A}$.

It is immediately clear that cocomplete spaces are subcomplete. Although from the definitions one might expect that these two properties are quite similar, we shall prove later on that they have an essentially different behaviour.

That both concepts are generalizations of topological completeness of metrizable spaces follows from the following theorem. 
2.3. THEOREM. For metrizable spaces the following properties are equivalent
(1) topological completeness
(2) cocompleteness
(3) subcompleteness

Proof. (1) $\Rightarrow(2)$ Let $X$ be a metrizable space and let $d$ be a metric for which $X$ is complete. $\mathbb{N}_{0}=\mathbb{N} \backslash\{0\}$.

For $i \in \mathbb{N}_{0}$ we consider an open cover $\mathscr{O}_{i}$ of $X$ with balls of radius $r \leqq 1 / i$. Then there exists a locally finite open refinement $\mathscr{P}_{1}$ of $\mathscr{O} i$. As a consequence of a result of Dowker [9] we can find a locally finite open refinement $\mathscr{A}_{1}$ of $\mathscr{P}_{1}$ with the property that any subfamily of $\mathscr{A}_{i}$ with the finite intersection property is finite.

Then $\mathscr{A}=\cup_{i \in N_{1}} \mathscr{A}_{i}$ is a pointbase for $X$ and for $\mathscr{C} \subset \mathscr{A}$ with the finite intersection property we have that $\alpha[\mathscr{C}] \neq \varnothing$ since $(X, d)$ is complete.

(2) $\Rightarrow$ (3) trivial.

(3) $\Rightarrow(1)$ Let $(X, d)$ be a metric space and $\mathscr{A}$ a pointbase with the property that every filterbase in $\mathscr{A}$ has a nonempty adherence.

We consider $\tilde{X}$, the completion of $X$ in the metric $d$. We shall prove that $X$ is a $G_{\delta}$ subset of $\tilde{X}$. Then by a wellknown theorem of Alexandroff and Hausdorff $(X, d)$ will be topological complete [10].

For $i \in \mathbb{N}_{0}$ we put $\mathscr{A}_{1}=\{A \mid A \in \mathscr{A}, \delta(A)<1 / i\}$ where $\delta$ indicates the $d$-diameter.

We consider $O_{i}=\cup\left\{\tilde{V} \mid \tilde{V}\right.$ is open in $\tilde{X}$ and there exists $A \in \mathscr{A}_{i}$ with $A \supset \tilde{V} \cap X\}$.

Then we clearly have that $X \subset \cap_{i \in N_{0}} O_{\imath}$ (Proposition 1.2).

Now take $y \in \bigcap_{t \in N_{i}} O_{i}$.

For $i \in \mathbb{N}_{0}$ we choose $A_{\imath} \in \mathscr{A}_{\imath}$ and $\tilde{V}_{i}$ open in $\tilde{X}$ such that $\tilde{V}_{\imath} \cap X \subset$ $A_{\imath}$ and $y \in \tilde{V}_{\text {r. }}$.

We shall write ${ }^{-}$for the closure in $\tilde{X}$ and ${ }^{-x}$ for the closure in $X$.

Now from the density of $X$ in $\tilde{X}$ and the openness of $\tilde{V}_{1}$ we have $\vec{V}_{\imath} \cap X=\vec{V}_{\imath} \subset \bar{A}_{\imath}$ and therefore $y \in \bar{A}_{\imath}$ for each $i \in \mathbb{N}_{0}$. It easily follows that $\left\{A_{l} \mid i \in \mathbb{N}_{0}\right\}$ is a filterbase.

Consequently there exists a point $x \in X$ with $x \in \bigcap_{i \in N_{0}} \bar{A}_{i}^{X} \subset$ $\bigcap_{\iota \in \mathbb{N}_{0}} \bar{A}_{t}$ which implies $y=x$.

3. Basic properties. In the statement of the next theorem we use the expression "locally compact, except possibly in one point". By this we mean that with the exception of at most one point, for each point $x$ and each filter $\mathscr{F}$, which converges to $x, \mathscr{F}$ contains a compact set. 
A subset $A$ of a convergence space $X$ is called relatively compact if every ultrafilter containing $A$ is convergent.

3.1. THEOREM. A convergence space which is locally compact, except possibly in one point, is cocomplete and therefore also subcomplete.

Proof. Let $X$ be a locally compact space except possibly in $x \in$ $X$. Consider the family $\mathscr{A}=\{A \mid A$ relatively compact $\} \cup \dot{x}$. Then for $y \neq x$ we consider the filterbase

$$
\mathscr{A}(y)=\{A \mid y \in A \text { and } A \text { relatively compact }\}
$$

Because of the local compactness for a filter $\mathscr{F}$ converging to $y$ we clearly have that $\mathscr{F} \cap \mathscr{A}(y)$ is a filterbase and that $[\mathscr{F} \cap \mathscr{A}(y)]=\mathscr{F} \cap \dot{y}$ which converges to $y$.

For $x$ we let $\mathscr{A}(x)=\dot{x}$.

Then it is clear that $\mathscr{A}$ is a pointbase making $X$ cocomplete.

3.2. THEOREM. The product of an arbitrary family of cocomplete (subcomplete) convergence spaces is cocomplete (subcomplete).

Proof. Let $\left(X_{i}\right)_{i \in I}$ be a family of spaces such that for $i \in I X_{i}$ is cocomplete (subcomplete) with respect to a pointbase $\mathscr{A}_{i}$.

On $\Pi_{i \in I} X_{i}$ consider the family $\mathscr{A}$ consisting of products $\Pi_{i \in I}^{\prime} A_{i}$ with $A_{i} \in \mathscr{A}_{i}$ and $A_{i}=X_{i}$ for all but a finite number of indices. Then it is easily verified that $\mathscr{A}$ is a pointbase making $\prod_{i \in I} X_{\imath}$ cocomplete (subcomplete).

3.3. That cocompleteness nor subcompleteness are invariant under convergence quotient maps follows from an example of Michael and Stone [20]. A mapping $f: \mathbb{P} \rightarrow \mathbb{Q}$ from the irrationals to the rationals is constructed which is a topological quotient map fulfilling the condition that for $y \in \mathbb{Q}$ there is an $x \in f^{-1}(y)$ such that $f(U)$ is a neighborhood of $y$ in $\mathbb{Q}$ iff $U$ is a neighborhood of $x$ in $\mathbb{P}$. It follows from [17] that $f$ is a convergence quotient map. $\mathbb{P}$ is cocomplete and therefore also subcomplete. $\mathbb{Q}$ has none of the two properties.

3.4. THEOREM. The sum of an arbitrary family of cocomplete (subcomplete) convergence spaces is cocomplete (subcomplete).

Proof. Let $\left(X_{i}\right)_{i \in I}$ be a family of spaces such that for $i \in I X_{i}$ is cocomplete (subcomplete) with respect to a pointbase $\mathscr{A}_{i}$ for $X_{i}$.

Let $X_{i}^{\prime}=X_{i} \times\{i\}$ and $X=\cup_{i \in I} X_{i}^{\prime}$ with the sumstructure $q$. Then it is easily checked that the family $\mathscr{A}=\cup_{i \in I} \mathscr{A}_{i}$ is a pointbase making $(X, q)$ cocomplete (subcomplete). 
3.5. THEOREM. For regular convergence spaces cocompleteness (subcompleteness) is open hereditary.

Proof. Let $X$ be regular and cocomplete with respect to a pointbase $\mathscr{A}$ and let $Y$ be an open subset.

Consider the family $\mathscr{B}=\{A \mid A \in \mathscr{A}, \bar{A} \subset Y\}$. For $y \in Y \mathscr{B}(y)=$ $\{A \mid A \in \mathscr{A}(y), \bar{A} \subset Y\}$ is a filterbase with $\mathscr{B}(y) \subset \dot{y}$.

For a filter $\mathscr{F}$ on $Y$ converging to $y$ in $Y$ we have that $[\mathscr{F}] \cap \mathscr{A}(y)$ is a filterbase and since $X$ is regular $\overline{[[\mathscr{F}] \cap \mathscr{A}(y)]}$ converges to $y$ in $X$.

$Y$ is open and therefore there is an $A \in[\mathscr{F}] \cap \mathscr{A}(y)$ with $\bar{A} \subset Y$. It follows then that $\mathscr{F} \cap \mathscr{B}(y)$ is a filterbase and that $[[\mathscr{F}] \cap \mathscr{A}(y)] \subset$ $[\mathscr{F} \cap \mathscr{B}(y)]$.

So $\mathscr{B}$ is a pointbase for $Y$.

If $\mathscr{C} \subset \mathscr{B}$ has the finite intersection property (is a filterbase) then $\varnothing \neq \alpha_{X}[\mathscr{C}] \subset \cap_{C \in \mathscr{C}} \bar{C} \subset Y$.

Since $Y \in[\mathscr{C}]$ it follows that $\alpha_{Y}[\mathscr{C}] \neq \varnothing$.

Without the regularity the previous theorem is not true since one can construct a cocomplete topology on $\mathbb{R}$ containing the rationals as an open subspace, cfr. [1].

3.6. None of the completeness properties is closed hereditary since the rationals can be closely embedded in the continuous product of real lines [10].

3.7. We now give an example showing that the completeness properties are not invariant under the coreflectors $\psi$ and $\lambda$ from the category of convergence spaces to the category of pretopological and topological spaces respectively.

Counterexample. We consider the rationals $\mathbb{Q}$ with the usual topo$\operatorname{logy} q$. Then we take the locally compact structure $p$ associated with $q$ as described in [18]. Since $(\mathbb{Q}, q)$ is a $k$-space we have $\lambda p=q$. It is easily checked that in this example we also have $\psi p=q$ since the closure operators of $p$ and $q$ coincide.

$(\mathbb{Q}, p)$ is cocomplete and subcomplete but $(\mathbb{Q}, q)$ has none of the completeness properties.

4. Differences between the completeness properties. We shall now discuss the differences between cocompleteness and subcompleteness by exhibiting some properties possessed by one but not by the other. 
Given a pointbase $\mathscr{A}$ we can close it for finite unions $\left(\mathscr{A}^{v}\right)$ or for finite intersections $\left(\mathscr{A}^{\wedge}\right)$.

The question now is whether a space which is cocomplete (subcomplete) with respect to $\mathscr{A}$ is still cocomplete (subcomplete) with respect to $\mathscr{A}^{\vee}$ or $\mathscr{A}^{\wedge}$.

4.1. THEOREM. If a convergence space $X$ is cocomplete with respect to a pointbase $\mathscr{A}$ then it is still cocomplete with respect to the pointbase $\mathscr{A}^{\vee}$.

Proof. Let $X$ be cocomplete with respect to a pointbase $\mathscr{A}$ and let $\mathscr{C} \subset \mathscr{A}^{v}$ be a family with the finite intersection property. Take an ultrafilter $\mathscr{U}$ finer than $\mathscr{C}$.

For an element $C \in \mathscr{C}$ we can find $A_{C} \in \mathscr{A} \cap \mathcal{U}$ such that $A_{C} \subset$ C. Then consider $\mathscr{D}=\left\{A_{C} \mid C \in \mathscr{C}\right\}$.

We have that $\mathscr{D} \subset \mathscr{A}$ and $\mathscr{D} \subset \mathscr{U}$. Consequently $\mathscr{D}$ has the finite intersection property and hence $\alpha[\mathscr{D}] \neq \varnothing$.

Since $[\mathscr{C}] \subset[\mathscr{D}]$ we have $\alpha[\mathscr{C}] \neq \varnothing$.

We can interpret the previous theorem as an Alexander subbase lemma for cocompleteness since cocompleteness is a kind of compactness condition on $\mathscr{A}$. That subcompleteness does not have this property follows from the next example.

Counterexample. Consider the set $X=] 0,1[$ with the discrete topology. We define subsets $V_{n}$ and $W_{n}$ for $n \geqq 2$ as follows

$$
\left.V_{n}=\right] 0, \frac{1}{n}\left[\cup\left\{1-\frac{1}{n}\right\} \quad \text { and } \quad W_{n}=\right] 1-\frac{1}{n}, 1\left[\cup\left\{\frac{1}{n}\right\} .\right.
$$

We consider the family $\mathscr{A}=\{\{x\} \mid x \in X\} \cup\left\{V_{n} \mid n \geqq 2\right\} \cup\left\{W_{n} \mid n \geqq\right.$ 2\}. Then clearly $\mathscr{A}$ is a pointbase for $X$. It is easily checked that all filterbases in $\mathscr{A}$ are principal and therefore have nonempty adherence.

Consequently $X$ is subcomplete with respect to $\mathscr{A}$. Now consider $\mathscr{A}^{\vee}$ and $\left.\mathscr{B}=\left\{V_{m} \cup W_{m} \mid m \geqq 2\right\} \subset \mathscr{A}^{\vee} . \quad V_{m} \cup W_{m}=10,1 / m\right] \cup$ $\left[1-1 / m, 1\left[\right.\right.$, hence $\mathscr{B}$ is a filterbase in $\mathscr{A}^{v}$. But clearly we have $\alpha[\mathscr{B}]=\cap \mathscr{B}=\varnothing$. Hence $X$ is not subcomplete with respect to $\mathscr{A}^{v}$.

4.2. THEOREM. If a convergence space $X$ is cocomplete with respect to a pointbase $\mathscr{A}$ then it is still cocomplete with respect to $\mathscr{A}^{\wedge}$.

Proof. Trivial.

Again for subcompleteness this result is not true. If it were subcompleteness would imply cocompleteness and this is not the case as follows from an example mentioned in 4.4. 
Yet there is an advantage in working with subcompleteness as we shall show now.

Let $P$ be a property. We say that a space $X$ has $P$ locally if $X$ has an open cover with subsets having the property $P$. The question now is whether a space is cocomplete (subcomplete) if it has this property locally.

4.3. THEOREM. If a convergence space has the property of subcompleteness locally then it is subcomplete.

Proof. Let $(X, q)$ be a convergence space and $\mathcal{U}$ an open cover of $X$ such that for each $U \in U$ the space $\left(U, q_{U}\right)$ is subcomplete with respect to a pointbase $\mathscr{A}_{U}$.

We may assume that $\mathcal{U}$ is well ordered by $\leqq$ Consider the family

$\mathscr{A}=\left\{A \mid A \in \bigcup_{U \in \mathscr{U}} \mathscr{A}_{U}\right.$ for which we can find $U \in \mathcal{U}$ with $A \in \mathscr{A}_{U}$ and $A \not \subset U^{\prime}$ if $\left.U^{\prime} \lessgtr U\right\}$.

For $x \in X$ define $U_{0}=\min \{U \mid x \in U\}$ and take $\mathscr{A}(x)=\mathscr{A}_{U_{0}}(x)$. It easily follows that $\mathscr{A}$ is a pointbase for $(X, q)$.

Now let $\mathscr{B} \subset \mathscr{A}$ be a filterbase.

Define $U_{0}=\min \left\{U \mid \mathscr{A}_{U} \cap \mathscr{B} \neq \varnothing\right\}$ and choose $B_{0} \in \mathscr{B} \cap \mathscr{A}_{U_{0}}$.

Consider $\mathscr{B}_{0}=\left\{B \mid B \in \mathscr{B}, B \subset B_{0}\right\}$. We shall prove that $\mathscr{B}_{0} \subset \mathscr{A}_{U_{0}}$.

Take $B \in \mathscr{B}_{0}$. Then $B \in \mathscr{A}$ and hence we have $U_{1} \in \mathcal{U}$ with $B \in \mathscr{A}_{U_{1}}$ and $B \not \subset U$ if $U \lessgtr U_{1}$.

If $U_{1} \lessgtr U_{0}$ we have $\mathscr{A}_{U_{l}} \cap \mathscr{B}=\varnothing$ which is impossible.

If $U_{1} \supsetneqq U_{0}$ then $B \not \subset U_{0}$ which again is impossible.

So we have $U_{1}=U_{0}$ and therefore $B \in \mathscr{A}_{U_{0}}$.

$\mathscr{B}_{0} \subset \mathscr{A}_{U_{0}}$ is a filterbase so consequently $\alpha\left[\mathscr{B}_{0}\right] \neq \varnothing$.

Since $\left[\mathscr{B}_{0}\right]=[\mathscr{B}]$ we have $\alpha[\mathscr{B}] \neq \varnothing$.

4.4. That cocompleteness does not follow from the corresponding local property can be shown using an example of $\mathrm{M}$. Ellen Rudin. In [11] and [5] a space is constructed which locally is completely metrizable and therefore locally is cocomplete (and subcomplete). From the previous theorem it follows that globally the space is subcomplete. But it can be shown not to be cocomplete [19].

5. Complete extensions. If $X$ is a convergence space we call a space $Y$ together with a mapping $f: X \rightarrow Y$ a cocomplete (subcomplete) extension of $X$ if $Y$ is cocomplete (subcomplete) and $f(X)$ is densely embedded in $Y$. 
Given a space $X$ and a pointbase $\mathscr{A}$ we shall construct a cocomplete extension $\rho(X, \mathscr{A})$ which will then also be subcomplete.

5.1. Construction. Let $(X, q)$ be a convergence space with pointbase $\mathscr{A}$. We can always suppose that $\mathscr{A}$ is closed for finite intersections.

Let $\phi(\mathscr{A})$ be the set of all families $\mathscr{C} \subset \mathscr{A}$ with the finite intersection property. This set, ordered by inclusion is inductive.

We define $Y=X \cup\{\mathscr{C} \mid \mathscr{C}$ is maximal in $\phi(\mathscr{A})$ and $\alpha[\mathscr{C}]=\varnothing\}$. For a subset $Z \subset X$ we define $\hat{Z}=Z \cup\{\mathscr{C} \mathscr{C} \in Y \backslash X, Z \in[\mathscr{C}]\}$ and for a filter $\mathscr{F}$ on $X$ we define $\hat{\mathscr{F}}=[\{\hat{F} \mid F \in \mathscr{F}\}]$.

We define a structure $p$ on $Y$ as follows.

A filter $\mathscr{H}$ on $Y$ converges to a point $x \in X$ iff there is a filter $\mathscr{F}$ on $X$ converging to $x$ in $q$ such that $\mathscr{F} \subset \mathscr{H}$. This is equivalent with the convergence of $\mathscr{\mathscr { H }}$ to $x$ in $q$, where $\mathscr{\mathscr { H }}=\{Z \mid Z \subset X, \hat{Z} \in \mathscr{H}\}$.

A filter $\mathscr{H}$ on $Y$ converges to $\mathscr{C} \in Y \backslash X$ iff $\mathscr{H} \supset[\hat{\mathscr{C}}]$. Then it is clear that $X$ is a dense subspace of $Y$.

Now consider the family $\hat{A}=\{\hat{A} \mid A \in \mathscr{A}\}$.

For $x \in X$ we have that $\hat{\mathscr{A}}(x)=\{\hat{A} \mid A \in \mathscr{A}(x)\}$ is a filterbase and for any filter $\mathscr{H}$, which converges to $x$ in $p$, we have that $\mathscr{H} \cap \hat{\mathscr{A}}(x)$ is a filterbase with

$$
[\mathscr{H} \cap \hat{\mathscr{A}}(x)]=[\check{\mathscr{H}} \cap \mathscr{A}(x)]^{\wedge}
$$

In $\mathscr{C} \in Y \backslash X$ we take $\hat{\mathscr{A}}(\mathscr{C})=\hat{\mathscr{C}}$.

So clearly $\hat{A}$ is a pointbase for $Y$.

If $\mathscr{D} \subset \hat{A}$ has the finite intersection property, then this is also the case for $\check{\mathscr{D}}=\{A \mid A \in \mathscr{A}, \hat{A} \in \mathscr{D}\}$. $\mathscr{\mathscr { D }} \in \phi(\mathscr{A})$ and hence we can find a maximal element $\mathscr{E}$ in $\phi(\mathscr{A})$ such that $\mathscr{D} \subset \mathscr{E}$.

In case $\alpha_{q}[\mathscr{E}]=\varnothing$ we have $\mathscr{D} \subset \hat{\mathscr{E}}$ and hence $\mathscr{E} \in \alpha_{p}[\mathscr{D}]$.

In case that there exists an $x \in \alpha_{q}[\mathscr{E}]$ and $\mathscr{F}$ is a filter converging to $x$ in $q$ and finer than $[\mathscr{E}]$ we have $\mathscr{F} \supset[\mathscr{D}]$ and therefore $x \in \alpha_{p}[\mathscr{D}]$.

So we have proved that $Y$ is cocomplete with respect to the pointbase $\hat{A}$. The space $(Y, p)$ constructed here is denoted $\rho(X, \mathscr{A})$.

\subsection{Proposition. If $X$ is a Hausdorff so is $\rho(X, \mathscr{A})$.}

Proof. This fact is based on the maximality of the elements $\mathscr{C}$ of $Y \backslash X$ and on the fact that $\alpha[\mathscr{C}]$ is empty.

If we take the pointbase $\mathscr{A}=2^{X}$ we obtain that $\rho(X, \mathscr{A})$ is the Stone Čech compactification of $X[21]$.

A natural question in this context is whether continuous mappings from a convergence space to a cocomplete (subcomplete) convergence space have continuous extensions to the completions. Since cocom- 
pleteness nor subcompleteness is closed hereditary, such a result can not be expected as follows from the following reasoning.

5.3. LemmA. If $X$ is a Hausdorff convergence space, $A$ a dense subset and $f$ a continuous mapping from $X$ to a convergence space $Y$ such that $f_{A}$ is a homeomorphism then we have $A=f^{-1}(f(A))$.

Proof. This result was proved for topological spaces in [14]. The proof can easily be rewritten using filter techniques.

5.4. Counterexample. As we mentioned earlier the set $\mathbb{Q}$ of the rationals is closely embedded in the continuous product $\mathbb{R}^{c}$ of real lines [10]. Consider the injection $f$ of $\mathbb{Q}$ into the complete space $\mathbb{R}^{c}$. For any completion $\overline{\mathbb{Q}}$ of $\mathbb{Q}$ the mapping $f$ has no extension to $\overline{\mathbb{Q}}$.

If $\overline{\mathrm{f}}: \overline{\mathbb{Q}} \rightarrow \mathbb{R}^{c}$ would be an extension of $f$ then $\bar{f}_{\mathrm{Q}}$ would be a homeomorphism. From the previous lemma it follows that $\bar{f}^{-1}(\mathbb{Q})=$ $\mathbb{Q}$. Consequently $\mathbb{Q}$ would be closed in $\overline{\mathbb{Q}}$ which is impossible.

\section{REFERENCES}

1. J. M. Aarts, Notes of colloquium cotopology, Mathematical Centre, Amsterdam 1965.

2. J. M. Aarts, Cocompactifications, Indagationes Math., 32, $\mathrm{n}^{\circ} 1$, (1970).

3. J. M. Aarts, J. de Groot and R. H. McDowell, Cocompactness, Nieuw Archief voor Wiskunde 3, XVIII, 1970.

4. - Cotopology for metrizable spaces, Duke Math. J., 37, (1970).

5. J. M. Aarts and E. Lowen-Colebunders, On an example of Mary Ellen Rudin. To appear.

6. J. M. Aarts and D. J. Lutzer, Completeness properties for recognizing Baire spaces, Dissertationes Mathematicae CXVI, 1974.

7. Bourbaki Nicolas, Eléments de Mathématique, Fas II. Topologie générale, Chap I, II.

8. C. H. Cook and H. R. Fisher, Uniform convergence spaces, Math. Annalen, 173, (1967).

9. C. H. Dowker, Mapping theorems for non-compact spaces, Amer. J. Math., 69, (1947).

10. R. Engelking, Outline of general topology, North-Holland Publ. C., Amsterdam 1968.

11. M E. Estill (Rudin), Concerning abstract spaces, Duke Math. J., 17, (1950).

12. H. R. Fisher, Limesräume. Math. Annalen, 137, 1959.

13. J. de Groot, Subcompactness and the Baire category theorem, Indagationes Math., 25, $\mathrm{n}^{\circ} 5$, (1963).

14. L. Gillman and H. Jerison, Rings of continuous functions, D. van Nostrand Inc., Princeton 1960.

15. H. Keller, Die Limes-Uniformisierbarkeit der Limesräume, Math. Annalen, 176, (1968).

16. D. C. Kent, Convergence functions and their related topologies, Fundam. Math., LIV, 1964.

17. - Convergence quotient maps, Fundam. Math. LXV, (1969).

18. D. C. Kent and G. D. Richardson, Locally compact convergence spaces. To appear.

19. E. Lowen-Colebunders, Completeness in convergence spaces. Thesis University Brussels, 1976

20. E. Michael and A. H. Stone, Quotient of the space of irrationals, Pacific J. Math., 28, (1969). 
21. G. D. Richardson, A Stone-Čech compactification for limit spaces, Proc Amer. Math. Soc., 25 (2), (1970).

22. Van der Slot, Some properties related to compactness, Thesis University of Amsterdam, (1968).

Received October 25, 1976 and in revised form February 21, 1977.

VRIJE UNIVERSITEIT BRUSSEL

PleinlaAn, 2, F7

AANGESTELD NAVORSER

B-1050 Brussel, Belgium

NFWO BELGIUM 



\section{PACIFIC JOURNAL OF MATHEMATICS \\ EDITORS}

RICHARD ARENS (Managing Editor)

University of California

Los Angeles, CA 90024

\section{R. A. Beaumont \\ University of Washington \\ Seattle, WA 98105}

C. C. MOORE

University of California

Berkeley, CA 94720

\section{J. DUGUNDJI}

Department of Mathematics

University of Southern California

Los Angeles, CA 90007

R. FINN AND J. MILGRAM

Stanford University

Stanford, CA 94305

\section{ASSOCIATE EDITORS}

\section{E. F. BECKENBACH}

B. H. NeUmanN

F. WOLF

K. YoSHIDA

\section{SUPPORTING INSTITUTIONS}

\author{
UNIVERSITY OF BRITISH COLUMBIA \\ CALIFORNIA INSTITUTE OF TECHNOLOGY \\ UNIVERSITY OF CALIFORNIA \\ MONTANA STATE UNIVERSITY \\ UNIVERSITY OF NEVADA \\ NEW MEXICO STATE UNIVERSITY \\ OREGON STATE UNIVERSITY \\ UNIVERSITY OF OREGON \\ OSAKA UNIVERSITY
}

\author{
UNIVERSITY OF SOUTHERN CALIFORNIA \\ STANFORD UNIVERSITY \\ UNIVERSITY OF HAWAII \\ UNIVERSITY OF TOKYO \\ UNIVERSITY OF UTAH \\ WASHINGTON STATE UNIVERSITY \\ UNIVERSITY OF WASHINGTON \\ AMERICAN MATHEMATICAL SOCIETY
}

The Supporting Institutions listed above contribute to the cost of publication of this Journal, but they are not owners or publishers and have no responsibility for its contents or policies.

Mathematical papers intended for publication in the Pacific Journal of Mathematics should be in typed form or offset-reproduced (not dittoed), double spaced with large margins. Underline Greek letters in red, German in green, and script in blue. The first paragraph or two must be capable of being used separately as a synopsis of the entire paper. Items of the bibliography should not be cited there unless absolutely necessary, in which case they must be identified by author and Journal, rather than by item number. Manuscripts, in duplicate, may be sent to any one of the four editors. Please classify according to the scheme of Math. Reviews, Index to Vol. 39. All other communications should be addressed to the managing editor, or Elaine Barth, University of California, Los Angeles, California, 90024.

100 reprints are provided free for each article, only if page charges have been substantially paid. Additional copies may be obtained at cost in multiples of 50 .

The Pacific Journal of Mathematics is issued monthly as of January 1966. Regular subscription rate: $\$ 72.00$ a year (6 Vols., 12 issues). Special rate: $\$ 36.00$ a year to individual members of supporting institutions.

Subscriptions, orders for back numbers, and changes of address should be sent to Pacific Journal of Mathematics, 103 Highland Boulevard, Berkeley, California, 94708.

PUBLISHED BY PACIFIC JOURNAL OF MATHEMATICS, A NON-PROFIT CORPORATION

Printed at Jerusalem Academic Press, POB 2390, Jerusalem, Israel.

Copyright (C) 1977 Pacific Journal of Mathematics All Rights Reserved 


\section{Pacific Journal of Mathematics \\ Vol. 70, No. $2 \quad$ October, 1977}

B. Arazi, A generalization of the Chinese remainder theorem ........... 289

Thomas E. Armstrong, Polyhedrality of infinite dimensional cubes .... . . . 297

Yoav Benyamini, Mary Ellen Rudin and Michael L. Wage, Continuous

images of weakly compact subsets of Banach spaces ............ 309

John Thomas Burns, Curvature functions on Lorentz 2-manifolds ......... 325

Dennis F. De Riggi and Nelson Groh Markley, Shear distality and equicontinuity .................................. 337

Claes Fernström, Rational approximation and the growth of analytic

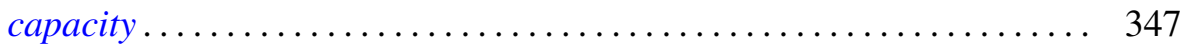

Pál Fischer, On some new generalizations of Shannon's inequality....... 351

Che-Kao Fong, Quasi-affine transforms of subnormal operators ......... 361

Stanley P. Gudder and W. Scruggs, Unbounded representations of

*-algebras........................................ 369

Chen F. King, A note on Drazin inverses .................... 383

Ronald Fred Levy, Countable spaces without points of first countability . . . 391

Eva Lowen-Colebunders, Completeness properties for convergence

spaces ......................................... 401

Calvin Cooper Moore, Square integrable primary representations ....... 413

Stanisław G. Mrówka and Jung-Hsien Tsai, On preservation of

E-compactness ................................ 429

Yoshiomi Nakagami, Essential spectrum $\Gamma(\beta)$ of a dual action on a von

Neumann algebra ................................ 437

L. Alayne Parson, Normal congruence subgroups of the Hecke groups

$G\left(2^{(1 / 2)}\right)$ and $G\left(3^{(1 / 2)}\right)$...

Louis Jackson Ratliff, Jr., On the prime divisors of zero in form rings . . . . 489

Caroline Series, Ergodic actions of product groups .................. 519

Robert O. Stanton, Infinite decomposition bases..................... 549

David A. Stegenga, Sums of invariant subspaces .................. 567 